\title{
A Model of Ultra-Short Pulsed Laser Ablation of Metal with Considering Plasma Shielding and Non-Fourier Effect
}

\author{
Sheng Tan *, Jianjun Wu *, Yu Zhang, Moge Wang and Yang Ou(D \\ College of Aerospace Science and Engineering, National University of Defense Technology, \\ Changsha 410073, China; zhangyu12@nudt.edu.cn (Y.Z.); wangmoge@nudt.edu.cn (M.W.); \\ ouyang16@nudt.edu.cn (Y.O.) \\ * Correspondence: tansheng17@nudt.edu.cn (S.T.); jjwu@nudt.edu.cn (J.W.); \\ Tel.: +86-0731-8457-5198 (S.T.); +86-0731-8457-5198 (J.W.)
}

Received: 14 October 2018; Accepted: 13 November 2018; Published: 15 November 2018

check for updates

\begin{abstract}
In this paper, a non-Fourier heat conduction model of ultra-short pulsed laser ablation of metal is established that takes into account the effect of the heat source, laser heating of the target, the evaporation and phase explosion of target material, the formation and expansion of the plasma plume, and interaction of the plasma plume with the incoming laser. Temperature dependent optical and thermophysical properties are also considered in the model due to the properties of the target will change over a wide range during the ultra-short pulsed laser ablation process. The results show that the plasma shielding has a great influence on the process of ultra-short pulsed laser ablation, especially at higher laser fluence. The non-Fourier effect has a great influence on the temperature characteristics and ablation depth of the target. The ultra-short pulsed laser ablation can effectively reduce the heat affected zone compared to nanosecond pulsed laser ablation. The comparison between the simulation results and the experimental results in the literature shows that the model with the plasma shielding and the non-Fourier effect can simulate the ultra-short pulsed laser ablation process better.
\end{abstract}

Keywords: ultra-short pulsed laser ablation; plasma shielding; non-Fourier effect; hyperbolic heat conduction equation; phase explosion; temperature-dependent material properties; ablation depth

\section{Introduction}

Ultra-short pulsed laser ablation has received significant attention recently due to their better control, high power density, low energy dissipation, smaller heat-affected area, minimal collateral material damage, lower ablation thresholds, and excellent mechanical properties $[1,2]$. Therefore, Ultra-short pulsed laser ablation is being used in a variety of applications such as nanoparticle manufacturing [3,4], micromachining [5-7], surface modification [8], laser ablation propulsion [9], laser-induced breakdown spectroscopy [10], laser welding [11], among many others. During the ultra-short pulsed laser ablation process, the interaction between the laser beam and the target material will result in the ablation of the target and consequently the formation of a crater on the target surface [12]. The two ablation mechanisms widely recognized by researchers are the evaporation and the pulse explosion [13].

Because the interaction time of ultra-short pulsed laser with material is very short $\left(\sim 10^{-13} \mathrm{~s}\right)$ and the heat-affected area is very small $\left(\sim 10^{-7} \mathrm{~m}\right)$ [14], it is difficult to study the relevant physical mechanism of ultra-short pulsed laser ablation by experiment. Therefore, ultra-short pulsed laser ablation has always been a research field in which mathematical models play an important role [14]. The heat conduction model based on the Fourier law can successfully deal with the most heat transfer 
problems, even though this model assumes that the velocity of the thermal wave propagation is infinite [15]. However, it can't accurately predict the heat conduction problems in some special cases, such as ultrafast heating, low-temperature condition and micro/nanoscale heat conduction [16]. In order to consider the fact that the velocity of the thermal wave propagation is finite, an improved theory called hyperbolic heat conduction was proposed to solve the heat conduction problems in these special cases [17].

Cattaneo [18] and Vernottee [19] were the first to propose the non-Fourier heat conduction equation by a hyperbolic heat conduction equation coupling with the local energy balance and the model was called the Cattaneo-Vernotte (CV) model. Vick and Ozisik provided an analytic solution in a semi-infinite medium containing volumetric energy sources by solving the hyperbolic heat conduction equation, and the result showed that the heat flow grows gradually with a relaxation time [20]. The single-phase-lagging (SPL) model is an extension of the CV model, which could convert into the CV model by doing the first-order Taylor approximation [21]. Tzou further introduced phase lags to the Fourier's law and gives a dual-phase-lag (DPL) model [22], and the validity of the model was verified by comparing with the experimental results [23]. Tang and Araki estimated the relaxation parameters and the thermal diffusivity by comparing the numerical results of the DPL model with the experimental results [22]. Subsequently, Tzou et al. coupled the temperature-dependent phase-lags into the DPL model to better agreement with the experimental result of ultra-short pulsed laser heating [24]. Zhang et al. solved the hyperbolic heat conduction equation with the heat source term, and the results showed that the non-Fourier effect can't be ignored and the heat source term has a significant effect on the non-Fourier heat conduction [25]. Chen proposed a hybrid method to solve the multidimensional hyperbolic heat conduction problem [26]. Ahmadikia et al. presented the analytical solutions of the non-Fourier heat conduction problem during laser irradiation of skin tissue [27]. Qi et al. established a Cattaneo-type time fractional non-Fourier heat conduction model with the heat source term for laser heating [15]. Zhang et al. solved the two-dimensional hyperbolic heat conduction equation when the local surface of a semi-infinite medium is irradiated by laser [17]. Kumar et al. used the finite element method to solve the three-dimensional DPL model for ultra-short pulsed laser heating [28]. Noroozi et al. considered the temperature-dependent thermal conductivity in the DPL model, and the results showed that the nonlinear analysis is significant in non-Fourier heat conduction problems [29]. Ma et al. presented an analytical solution for the CV wave model when the target is irradiated by a moving laser pulse [30]. Soares proposed an enhanced explicit technique to analyze hyperbolic heat transfer problems which could reduce computational costs [31].

In addition, there are many other models used to simulate the interaction of ultra-short pulsed laser with material [14]. Among them, the two-temperature model (TTM), which is also called a parabolic two-step model (PTSM), was more commonly used [32-35]. For a detailed description of the TTM, see References [14,36-38]. At present, after years of development, the TTM has proposed many improved models [39-46], such as dual-parabolic two-step model (DPTSM) [14], hyperbolic two-step model (HTSM) [39], dual-hyperbolic two-step model (DHTSM) [40], semiclassical two-temperature model (STTM) [42]. The successful application of DHTSM [47-52] also indicates that the effect of the hyperbolic effect or relaxation time cannot be ignored, especially when the laser pulse time is comparable to or shorter than the electron relaxation time [49]. At the same time, the method of simulating the interaction between laser and material by coupling the TTM with other methods has also been developed, such as the coupling of TTM and molecular dynamics method (TTM-MD) [53-56], the coupling of TTM and hydrodynamic model (TTM-HD) $[57,58]$, and the coupling of TTM and classical heat accumulation model [59]. Despite this, the limited validity of the TTM still exists [60-63], one of which is that the validity verification at higher laser fluence is still problematic [63]. In addition, the effects of plasma shielding are not considered in most TTMs, which may lead to overestimation of ablation depth at higher laser fluence $[64,65]$.

In this paper, a one-dimensional non-Fourier heat conduction model with the heat source term is established to investigate the target temperature profile and ablation depth when the metal target is 
irradiated by ultra-short pulsed laser. The model considers both evaporation and phase explosion, and also considers the plasma expansion and the interaction of the plasma plume with the incoming laser. In addition, to account the fact that the properties of the target would change greatly in a wide range of temperature from ambient temperature to close to the critical temperature, the temperature dependence of optical and thermophysical properties of aluminum (Al) are considered in the model. The finite volume method (FVM) is used to solve the hyperbolic heat conduction equation.

The paper is organized in sections as follows: Section 2 presents the process of establishing the theoretical model in detail. In Section 3, the effects of the plasma shielding, relaxation time, and laser fluence are discussed and the effectiveness of model is verified by comparing the simulation results with the experimental results. Finally, some conclusions are given in Section 4.

\section{Theoretical Model}

\subsection{Heat Conduction Equation for Two Different Stages}

When the metal target is ablated by ultra-short pulsed laser, the absorption depth of the target is much smaller than the diameter of the laser beam. Therefore, the heat conduction problem can be described by one-dimensional heat conduction equation along the direction of laser irradiation. At the same time, in the process of ultra-short pulsed laser ablation, the heat affected area is small, which makes the thickness of melting layer smaller [65]. Therefore, the effect of the dielectric layer is not considered in this model, which has a great influence on nanosecond laser ablation $[66,67]$. In order to consider the effects of target evaporation, phase explosion, plasma expansion and plasma shielding, the ablation process is divided into two stages: Before and after evaporation and the corresponding heat transfer model are established.

\subsubsection{Heat Conduction Equation before Evaporation}

When the target temperature is less than boiling temperature, the heat taken away by target evaporation and plasma shielding is not considered. Therefore, the conservation law of energy can be expressed as [68]:

$$
\rho(T) C_{p}(T) \frac{\partial(T)}{\partial t}=-\nabla \overrightarrow{\mathbf{q}}+\dot{S}
$$

where $\rho(T), C_{p}(T)$ are density and specific heat which vary with the temperature, respectively. $\overrightarrow{\mathbf{q}}$ is the heat flux vector. Here $\dot{S}$ is the heating term which is given by:

$$
\dot{S}=\alpha(T) \beta(T) I_{L}(t) \exp [-\alpha(T) x]
$$

where $\alpha(T)$ and $\beta(T)$ are absorption coefficient and surface absorptivity of the target which vary with the temperature, respectively. $I_{L}(t)$ is the laser intensity that reaches the target surface.

Combining Equation (1) with the CV model [14]:

$$
\overrightarrow{\mathbf{q}}+\tau_{0} \frac{\partial \overrightarrow{\mathbf{q}}}{\partial t}=-k(T) \frac{\partial T}{\partial x}
$$

The hyperbolic heat conduction equation could be obtained as follows:

$$
\rho(T) C_{p}(T) \frac{\partial T}{\partial t}+\tau_{0} \rho(T) C_{p}(T) \frac{\partial^{2} T}{\partial t^{2}}=\frac{\partial}{\partial x}\left(k(T) \frac{\partial T}{\partial x}\right)+\dot{S}+\tau_{0} \frac{\partial \dot{S}}{\partial t}
$$

where $k(T)$ is thermal conductivity which varies with the temperature and $\tau_{0}$ is the relaxation time and is estimated to lie between $10^{-14} \mathrm{~s}$ and $10^{-11} \mathrm{~s}$ for pure metals [69]. 
The corresponding initial condition and boundary condition are given by:

$$
\left\{\begin{array}{l}
\left.T(x, t)\right|_{t=0}=T_{0} \\
\left.k(T) \frac{\partial T(x, t)}{\partial x}\right|_{x=l}=0 \\
-\left.k(T) \frac{\partial T(x, t)}{\partial x}\right|_{x=0}=-\beta(T) I_{L}(t)+\varepsilon \sigma\left(T^{4}-T_{0}^{4}\right)
\end{array}\right.
$$

where $T_{0}$ is the room temperature ( $\left.300 \mathrm{~K}\right), l$ is the length of target, $\sigma$ and $\varepsilon$ are the Stefan-Boltzmann constant and the emissivity of target, respectively.

\subsubsection{Heat Conduction Equation after Evaporation}

When the target surface temperature is higher than boiling point, the effects of evaporation and plasma shielding will become noticeable. Therefore, the heat taken away by evaporation and plasma shielding should be considered and the conservation law of energy can be given by [68]:

$$
\rho(T) C_{p}(T) \frac{\partial(T)}{\partial t}=-\nabla \overrightarrow{\mathbf{q}}+\dot{S}+\rho(T) C_{p}(T) u(T) \frac{\partial T}{\partial x}
$$

Combining Equation (6) with Equation (3), the hyperbolic heat conduction equation could be obtained as follows:

$$
\begin{aligned}
& \rho(T) C_{p}(T) \frac{\partial T}{\partial t}+\tau_{0} \rho(T) C_{p}(T) \frac{\partial^{2} T}{\partial t^{2}}= \\
& \frac{\partial}{\partial x}\left(k(T) \frac{\partial T}{\partial x}\right)+\dot{S}+\tau_{0} \frac{\partial \dot{S}}{\partial t}+\rho(T) C_{p}(T) u(T) \frac{\partial T}{\partial x}+\tau_{0} \frac{\partial}{\partial t}\left(\rho(T) C_{p}(T) u(T) \frac{\partial T}{\partial x}\right)
\end{aligned}
$$

where $u(T)$ is the velocity of evaporation which is given by [70,71]:

$$
u(T)=C_{s} P_{b}\left(2 \pi m_{a} k_{B} T_{s}\right)^{-1 / 2} \frac{m_{a}}{\rho\left(T_{s}\right)} \exp \left[\frac{L_{h v} m_{a}}{k_{B}}\left(\frac{1}{T_{b}}-\frac{1}{T_{s}}\right)\right]
$$

where $C_{s}$ is the evaporation coefficient, $P_{b}$ is the boiling pressure (100,000 Pa), $m_{a}$ is the mass of aluminum atom, $k_{B}$ is the Boltzmann constant, $T_{s}$ is the temperature of the target surface which varies with time, $T_{b}$ is the boiling point, $\rho\left(T_{s}\right)$ is the density which varies with the surface temperature, and $L_{h v}$ is the latent heat of vaporization.

Based on continuous temperature condition, the initial conditions for the second phase are given by:

$$
\left\{\begin{array}{l}
T\left(0 \leq x \leq l, t=t_{b}^{+}\right)=T\left(0 \leq x \leq l, t=t_{b}^{-}\right) \\
\left.\frac{\partial T}{\partial t}\right|_{t=t_{b}^{+}}=\left.\frac{\partial T}{\partial t}\right|_{t=t_{b}^{-}}
\end{array}\right.
$$

where $t_{b}$ is the time for the surface to reach the boiling point.

Based on the law of conservation of energy, the boundary condition can be expressed as:

$$
\left\{\begin{array}{l}
\left.k(T) \frac{\partial T(x, t)}{\partial x}\right|_{x=l}=0 \\
-\left.k(T) \frac{\partial T(x, t)}{\partial x}\right|_{x=0}=-\beta(T) I_{L}(t)+L_{h v} \rho(T) u(T)+\varepsilon \sigma\left(T^{4}-T_{0}^{4}\right)
\end{array}\right.
$$

\subsection{Plasma Expansion and Plasma Shielding}

The evaporation and phase explosion form a plasma plume on the target surface, and then the plasma plume will expand and absorb part of the incident laser energy [68]. The laser wavelength used in this model is $800 \mathrm{~nm}$. Therefore, in the plasma shielding model, the absorption of laser energy by plasma plume only considers the inverse bremsstrahlung mechanism (IB). Other mechanisms such as photo-ionization and Mie absorption are ignored in the model due to the influence of other absorption 
mechanisms being smaller when the laser wavelength is higher [72]. $\alpha_{I B}$, the absorption coefficient of IB, is given by [73]:

$$
\alpha_{I B}=\frac{3.69 \times 10^{8} Z^{3} n_{i}^{2}}{T_{p}^{0.5} \omega^{3}}\left[1-\exp \left(-\frac{h \omega}{k_{B} T_{p}}\right)\right]
$$

where $Z$ is the average charge, $n_{i}$ is the ion density, $T_{p}$ is the plasma temperature, $\omega$ is the laser frequency, and $h$ is the Planck constant.

The ion number density could be obtained from the Saha equation which is expressed as [74]:

$$
\frac{n_{i}^{2}}{n_{0}} \approx 2.4 \times 10^{21} T_{p}^{1.5} \exp \left(-\frac{I P_{1}}{k_{B} T_{p}}\right)
$$

where $n_{0}$ and $I P_{1}$ are the number density of neutral atoms and the first ionization potential, respectively.

Assuming that $n_{0}$ is uniformly distributed in the length of plasma shielding, it can be expressed as:

$$
n_{0}=\frac{\rho(T)}{m_{a}} \frac{S_{a b}}{H_{p}}
$$

where $S_{a b}$ is the ablation depth, and $H_{p}$ is the length of plasma shielding which is given by:

$$
H_{p}=S_{a b}+\int_{t_{b}}^{t} v_{p} d t
$$

where $v_{p}$ is the plasma expansion speed.

The length of plasma shielding varies with the plasma expands outward, and the dynamic equation of plasma expansion is given by [73]:

$$
x_{p}(t)\left(\frac{1}{t} \frac{d x_{p}(t)}{d t}+\frac{d^{2} x_{p}(t)}{d t^{2}}\right)=\frac{k_{B} T_{p}}{m_{a}}
$$

where $x_{p}(t)$ is the plasma expansion distance. The plasma expansion speed can be given by:

$$
v_{p}=\frac{d x_{p}(t)}{d t}
$$

In the calculation, the initial velocity of the plasma is defined by [75]:

$$
v_{0}=\sqrt{8 k_{B} T_{b} / \pi m_{a}}
$$

Therefore, the intensity of laser reaching the target surface after absorbing part of the energy by the plasma plume can be given by:

$$
I_{L}(t)= \begin{cases}I_{0}(t) & t<t_{b} \\ I_{0}(t) \exp \left(-\int_{0}^{H_{p}} \alpha_{I B} d x\right) & t \geq t_{b}\end{cases}
$$

where $I_{0}(t)$ is the incident laser intensity which is given by [34]:

$$
I_{0}(t)=I_{\max }\left(\frac{t}{t_{\max }}\right)^{7} \exp \left[7\left(1-\frac{t}{t_{\max }}\right)\right]
$$

where $I_{\max }$ and $t_{\max }$ are the maximum laser intensity and the time to reach the maximum laser intensity, respectively. 
The relationship between the maximum laser intensity and laser fluence $\left(F_{\text {fluence }}\right)$ could be given by:

$$
I_{\max }=F_{\text {fluence }} / \int_{0}^{\infty}\left(\frac{t}{t_{\max }}\right)^{7} \exp \left[7\left(1-\frac{t}{t_{\max }}\right)\right] d t
$$

The ratio of the laser intensity to the maximum laser intensity varies with time is shown in Figure 1. The laser pulse time $\left(t_{p}\right)$ taken as full width at half maximum (FWHM) is also shown in Figure 1. In this paper, the pulse time of the laser set as $170 \mathrm{fs}$.

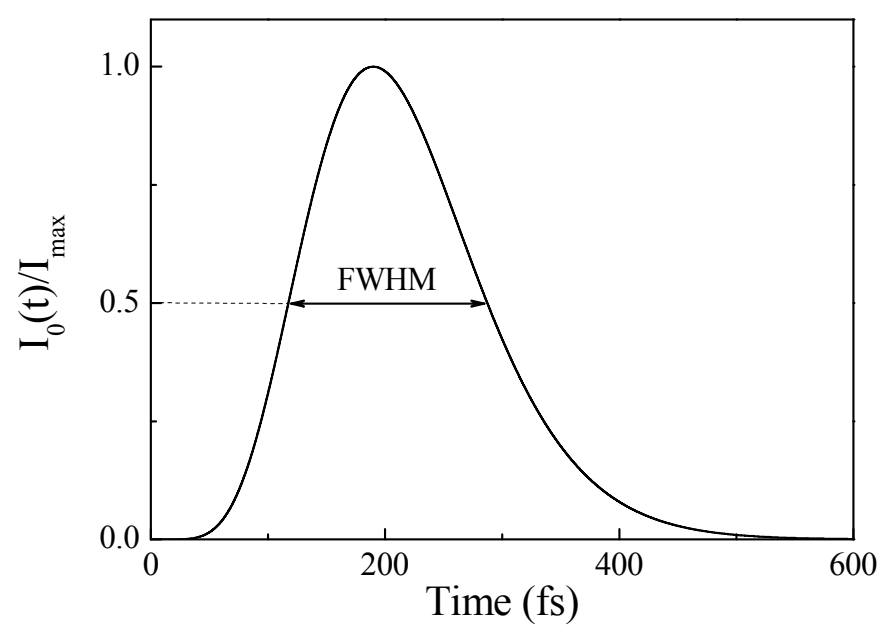

Figure 1. The variation of the ratio of laser intensity to maximum laser intensity with time $\left(t_{p}=170 \mathrm{fs}\right)$.

\subsection{Properties of $A l$}

The temperature dependence of optical and thermophysical properties and other parameters of Al used in this paper are summarized in Table 1.

Table 1. Parameters of aluminum (Al) used in the model.

\begin{tabular}{|c|c|c|c|c|c|}
\hline Parameters & Symbols & \multicolumn{3}{|c|}{ Values } & Reference \\
\hline Melting point/K & $T_{m}$ & \multicolumn{3}{|c|}{933.47} & {$[76]$} \\
\hline Boiling point/K & $T_{b}$ & \multicolumn{3}{|c|}{2792.15} & {$[76]$} \\
\hline $\begin{array}{c}\text { Latent heat of } \\
\text { vaporization } /(\mathrm{J} / \mathrm{kg})\end{array}$ & $L_{h v}$ & \multicolumn{3}{|c|}{$1.05 \times 10^{7}$} & {$[76]$} \\
\hline $\begin{array}{l}\text { First ionization } \\
\text { potential } / \mathrm{eV}\end{array}$ & $I P_{1}$ & \multicolumn{3}{|c|}{5.98} & {$[76]$} \\
\hline Critical temperature/K & $T_{c r}$ & \multicolumn{3}{|c|}{8944.00} & [77] \\
\hline Evaporation coefficient & $C_{s}$ & \multicolumn{3}{|c|}{0.82} & [67] \\
\hline $\begin{array}{c}\text { Electrical } \\
\text { conductivity/(S/m) }\end{array}$ & $\sigma(T)$ & $\sigma(T)=\left\{\begin{array}{l}1 \\
1 \\
2\end{array}\right.$ & $\begin{array}{l}10^{8} /(0.0122 T-1.0071) \\
10^{8} /(0.0136 T+12.473) \\
29,000\end{array}$ & $\begin{array}{r}T<T_{m} \\
T_{m} \leq T<0.8 T_{c r} \\
T \geq 0.8 T_{c r}\end{array}$ & {$[77,78]$} \\
\hline $\begin{array}{c}\text { Thermal } \\
\text { conductivity } /(\mathrm{W} /(\mathrm{m} \cdot \mathrm{K}))\end{array}$ & $k(T)$ & {$[k(T)=$} & $\begin{cases}226.93+0.03 T & T \\
263.29-0.06 T & 40 \\
2.44 \times 10^{-8} \sigma(T) T & T\end{cases}$ & $\left.\begin{array}{l}<400 \mathrm{~K} \\
00 \mathrm{~K} \leq \mathrm{T}<T_{m} \\
\geq T_{m}\end{array}\right]$ & {$[76]$} \\
\hline Density/(kg/m³ $)$ & $\rho(T)$ & {$[\rho(T)=$} & $=\left\{\begin{array}{r}2863.25464-0.52305 \\
2592.49851-0.233 T\end{array}\right.$ & $\left.\begin{array}{ll}5 T & T<T_{m} \\
T & T \geq T_{m}\end{array}\right]$ & {$[76]$} \\
\hline
\end{tabular}


Table 1. Cont.

\begin{tabular}{cccc}
\hline Parameters & Symbols & Values & Reference \\
\hline Specific heat $/(\mathrm{J} /(\mathrm{kg} \cdot \mathrm{K}))$ & $C_{p}(T)$ & {$\left[C_{p}(T)=\left\{\begin{array}{lll}762+0.467 T & T<T_{m} \\
921 & T \geq T_{m}\end{array}\right]\right.$} & {$[76]$} \\
\hline refractive index & $n_{R}(T)$ & The calculation equation is shown in Reference [71] & {$[71]$} \\
\hline extinction coefficient & $n_{I}(T)$ & The calculation equation is shown in Reference [71] & {$[71]$} \\
\hline $\begin{array}{c}\text { Absorption } \\
\text { coefficient/ }(1 / \mathrm{m})\end{array}$ & $\alpha(T)$ & $\alpha(T)=\frac{4 \pi n_{I}(T)}{\lambda}$ & {$[71]$} \\
\hline Absorptivity & $\beta(T)$ & $\beta(T)=\frac{4 n_{R}(T)}{\left(n_{R}(T)+1\right)^{2}+n_{I}{ }^{2}(T)}$ & {$[71]$} \\
\hline
\end{tabular}

\subsection{Numerical Method}

The hyperbolic heat conduction equation is solved by using the finite volume method. In the numerical calculation, five parameters, i.e., density, specific heat, thermal conductivity, absorption coefficient and absorptivity, in every layer are updated in each time step. In the second stage, the plasma plume expansion and the laser intensity after passing through the plasma plume are calculated simultaneously, and coupled in each time step.

Ablation depth due to evaporation is calculated by using the evaporation velocity at each time step. In this paper, it is assumed that the target layers that reach $0.9 T_{c r}$ are immediately ablated by phase explosion, and these layers are accounted as explosion depth and are ignored in the subsequent calculation [71,72]. The ablation depth of the target is the sum of the evaporation depth and the phase explosion depth [71].

\section{Results and Discussions}

\subsection{The Effect of Plasma Shielding}

In this section, the effects of the plasma shielding are discussed. The variations of laser intensity and ablation depth, without and with plasma shielding, with time are compared in Figure 2 at laser fluence of $10 \mathrm{~J} / \mathrm{cm}^{2}$. The figure shows that the variation curves of laser intensity and ablation depth have great difference between with plasma shielding and without plasma shielding. The separation time of the two ablation depth curves is the same as that of the two laser intensity curves. This is because when the ablation depth reaches a certain level, dense plasma will be generated on the target surface, which reduces the laser intensity that reaches the target surface. This, in turn, affects the ablation process and slows down the increase of the ablation depth.

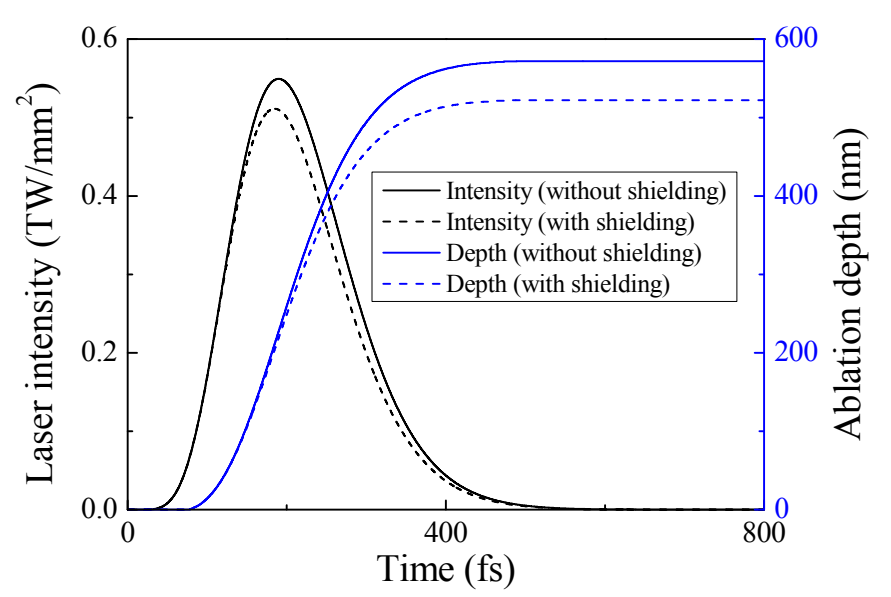

Figure 2. A comparison of laser intensity and ablation depth $\left(F_{\text {fluence }}=10 \mathrm{~J} / \mathrm{cm}^{2}\right)$.

Figure 3 shows the variation of plasma shielding proportion, which is the ratio of fluence absorbed by plasma plume to fluence of incident laser fluence, with laser fluence. The figure shows that the 
shielding proportion increases as the laser fluence increases which indicates that the opacity of the plasma plume increases with the increase of the laser fluence. Therefore, it can be concluded that the plasma shielding can't be ignored in the process of ultra-short pulsed laser ablation, especially at high laser fluence. The effect of the plasma shielding is considered in the subsequent results presented in the paper.

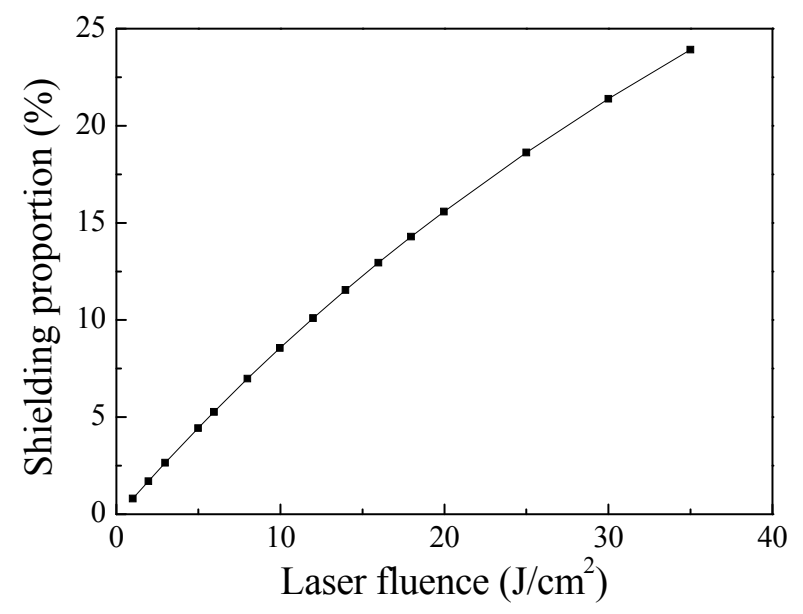

Figure 3. Variation of plasma shielding proportions with laser fluence.

\subsection{The Effect of Relaxation Time}

In this section, the effects of the relaxation time are discussed. The temperature variation in the surface layer of target at different relaxation time is shown in Figure 4 for a laser fluence of $10 \mathrm{~J} / \mathrm{cm}^{2}$. As can be seen from the figure, the relaxation time has a notable effect on the temperature. The rising time of temperature of surface layer becomes late as the relaxation time increases, and the rising rate of surface layer temperature becomes slow with the increase of relaxation time. At relaxation times of $0.0 \mathrm{~s}, 1.0 \times 10^{-14} \mathrm{~s}, 1.0 \times 10^{-13} \mathrm{~s}, 1.0 \times 10^{-12} \mathrm{~s}$, the time of the surface layer temperature rising to the melting point is $47.2 \mathrm{fs}, 51.9 \mathrm{fs}, 60.4 \mathrm{fs}$ and $67.3 \mathrm{fs}$, respectively. When the temperature exceeds the melting point, the surface layer temperature increases exponentially and the growth rate decreases with the increase of the relaxation time. Finally, the temperature of surface layer reaches $0.9 T_{c r}$ and the phase explosion ablation occurs.

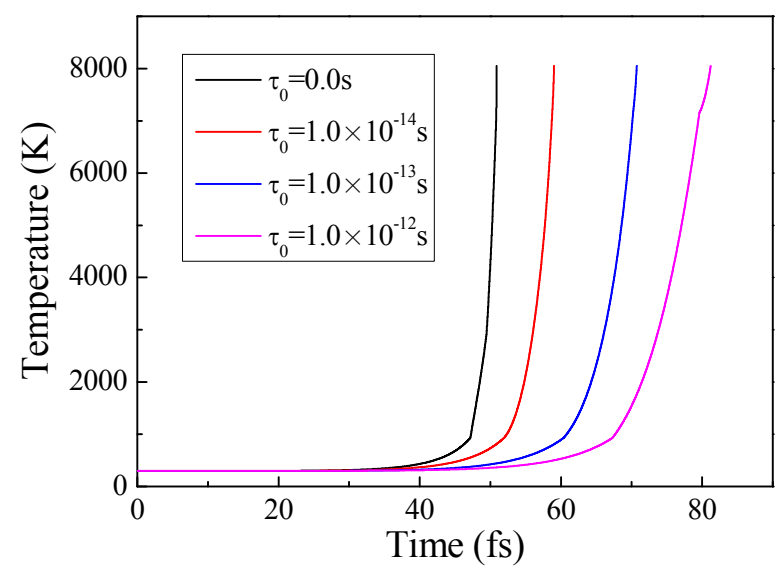

Figure 4. The temperature variation in the surface layer of target at different relaxation time $\left(F_{\text {fluence }}=10 \mathrm{~J} / \mathrm{cm}^{2}\right)$.

The surface temperature characteristics of the target are shown in Figure 5 under different relaxation times. It should be noted that the target surface refers to the instantaneous surface position after ablation, which varies with the ablation depth. As can be seen from the figure, for the same 
relaxation time, the surface temperature of the target rises rapidly to $0.9 T_{c r}$ and induces the phase explosion. Because of the continuous injection of laser energy, the phase explosion will continue to occur, so the temperature will be maintained at the position of $0.9 T_{c r}$. When the laser energy is reduced to a certain value, the surface temperature cannot be maintained at $0.9 T_{c r}$, which means that the phase explosion ends, and the target temperature will begin to drop. When the target temperature drops to $0.8 T_{c r}$, the optical and thermophysical properties (absorptivity, absorption coefficient, thermal conductivity) of the target will change greatly at this temperature, so there will be a small range of sudden drop in the target temperature.

Compared with the different relaxation time, the relaxation time has a notable effect on the temperature characteristics of the target surface. When the relaxation time is 0.0 s, i.e., without considering the non-Fourier effect, the time of surface temperature rising to $0.9 T_{c r}$ is the fastest and the end of the phase explosion is at the latest which means that the maintenance time of phase explosion is the longest. When the relaxation time is higher than 0.0 s, i.e., with considering the non-Fourier effect, the maintenance time of phase explosion decreases as the relaxation time increases.

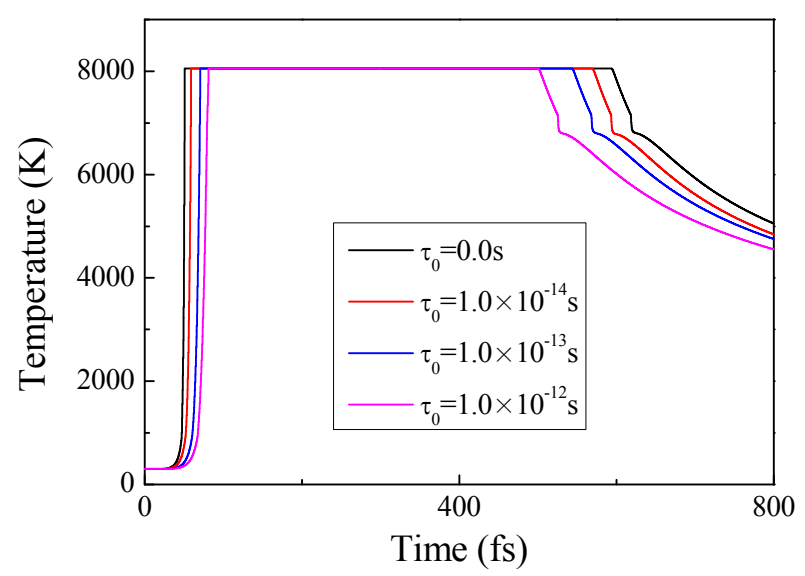

Figure 5. The surface temperature characteristics of the target under different relaxation times $\left(F_{\text {fluence }}=10 \mathrm{~J} / \mathrm{cm}^{2}\right)$.

The ablation depths vary with time at different relaxation times are shown in Figure 6. The slops of the ablation depth indicate that the ablation rate initially increases and then the rate of increase slows down gradually until it equals to zero. At the same time, the relaxation time has a notable effect on the ablation depth of the target, and when the relaxation time is $0.0 \mathrm{~s}$, the ablation depth is the deepest. However, when the relaxation time is higher than zero, the ablation depth is shallower with the increase of the relaxation time, but the trend of shallower ablation depth decreases as the relaxation time increases.

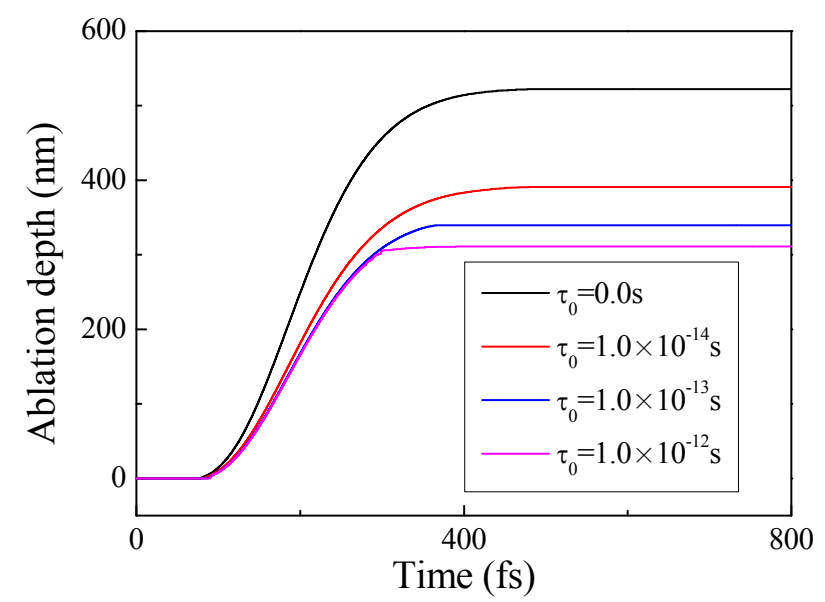

Figure 6. The ablation depths vary with time at different relaxation times $\left(F_{\text {fluence }}=10 \mathrm{~J} / \mathrm{cm}^{2}\right)$. 


\subsection{The Effect of Laser Fluence}

The temperature characteristics of four different laser fluence with at relaxation time of $1.0 \times 10^{-13} \mathrm{~s}$ are shown in Figure 7. As shown in this figure, as the laser fluence increases, the time at which the surface of the target reaches the phase explosion is advanced, and the time at which the phase explosion ends is correspondingly delayed, thereby the maintenance time of phase explosion increases.

Figure 8 shows the variation of ablation depth with time when the relaxation time is $1.0 \times 10^{-13} \mathrm{~s}$ under four different laser fluences. As shown in the figure, when the ablation depth is increasing, the slope of the ablation depth curve increases as the laser fluence increases which indicates that the ablation rate is faster. Therefore, the ablation depth increases with the increase of the laser fluence. At the same time, it can be seen from the figure that the biggest difference in ablation depth under different laser fluences is at the FWHM of the laser, and the difference of ablation depth caused by other time is small. In addition, although the evaporation ablation rate is faster at the phase explosion temperature (about $79 \mathrm{~m} / \mathrm{s}$ ), the evaporation depth is small due to the short duration. Even when the fluence is $20 \mathrm{~J} / \mathrm{cm}^{2}$, the evaporation depth is only $0.05 \mathrm{~nm}$. This indicates that the ablation mechanism of ultra-short pulsed laser ablation is dominated by phase explosion. However, in nanosecond laser ablation, there is a laser fluence threshold which changes from evaporation to phase explosion [71].

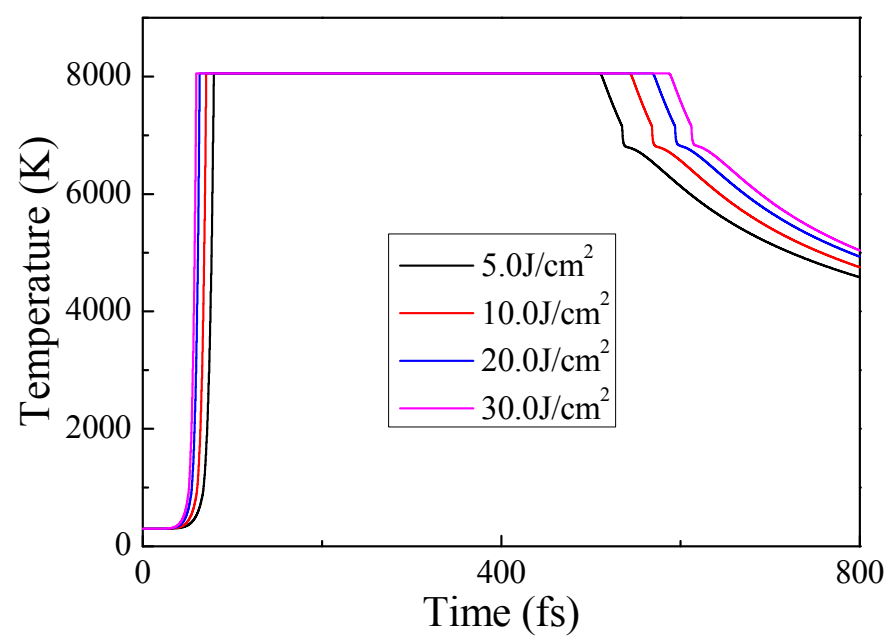

Figure 7. The temperature characteristics under four different laser fluence $\left(\tau_{0}=1.0 \times 10^{-13} \mathrm{~s}\right)$.

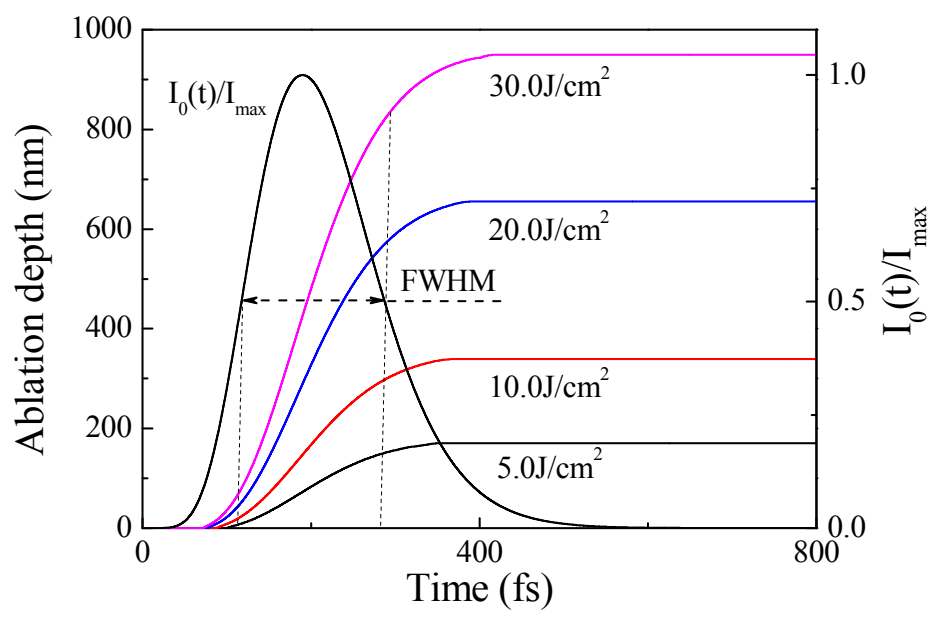

Figure 8. The ablation depths vary with time under four different laser fluence $\left(\tau_{0}=1.0 \times 10^{-13} \mathrm{~s}\right)$. 
The ablation depth, the superheated liquid layer (temperature above the boiling point and below $0.9 T_{c r}$ ) and the melting layer (temperature above the melting point and below the boiling point) as a function of time are shown in Figure 9. It can be seen from the figure that the thickness of the melting layer and the superheated liquid layer are very small during the period from the beginning to the end of the ablation, which indicates that the ablation speed of ultra-short pulsed laser is very fast and the heat conduction to the inside of the target is less. After the ablation is finished, the depth of the melted layer and the superheated liquid layer will increase due to the large temperature gradient on the surface and inside of the target, but the depth of the increase is lower, and the sum of the depths of the two is less than $30 \mathrm{~nm}$. In the Reference [71], the melting depth of the nanosecond laser ablation ( $F_{\text {fluence }} \approx 17 \mathrm{~J} / \mathrm{cm}^{2}, t_{p}=10 \mathrm{~ns}$ ) is more than $2000 \mathrm{~nm}$, which indicates that ultra-short pulsed laser ablation can effectively reduce the heat affected zone compared to nanosecond pulsed laser ablation.

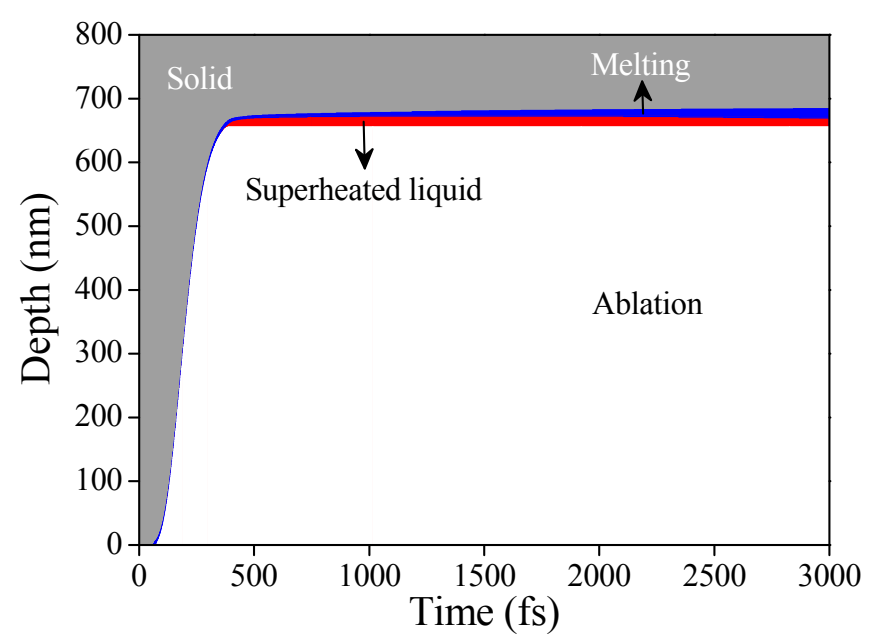

Figure 9. The ablation depth, the superheated liquid layer and the melting layer as a function of time $\left(F_{\text {fluence }}=20 \mathrm{~J} / \mathrm{cm}^{2}\right)$.

\subsection{Model Validation}

In order to verify the validity of the model, numerical simulations were carried out under the same conditions as the experiments in Reference [58]. At the same time, although the simulation results of ultra-short pulsed laser ablation of Al by TTM-HD in Reference [58] agree well with the experimental results, the model used in Reference [58] cannot study the ablation mechanism and does not consider the effect of plasma shielding, which cannot be ignored at higher laser fluence.

Figure 10 presents the experimental results and the simulation results of three theoretical models which are the model without considering plasma shielding and the non-Fourier effect, the model with considering plasma shielding but without considering the non-Fourier effect, and the model with considering plasma shielding and the non-Fourier effect, respectively.

It can be seen from Figure 10 that the simulation results are higher than the experimental results when the non-Fourier effect is not considered whether or not the plasma shielding is considered. The simulation results based on the model without considering plasma shielding are higher than the results based on the model with considering plasma shielding and the difference between the results of the two models increases as the laser fluence increases. This is because the proportion of laser fluence absorbed by plasma plume increases as the laser fluence increases.

On the contrary, the simulation results based on the model considering plasma shielding and the non-Fourier effect are in better agreement with the experimental results. Therefore, it can be concluded that the plasma shielding and the non-Fourier effect play an important role in the ultra-short pulsed laser ablation process. Although there are some differences between the results of simulation and experiment, the model with considering the plasma shielding and the non-Fourier effect can still simulate the ultra-short pulsed laser ablation process better. 


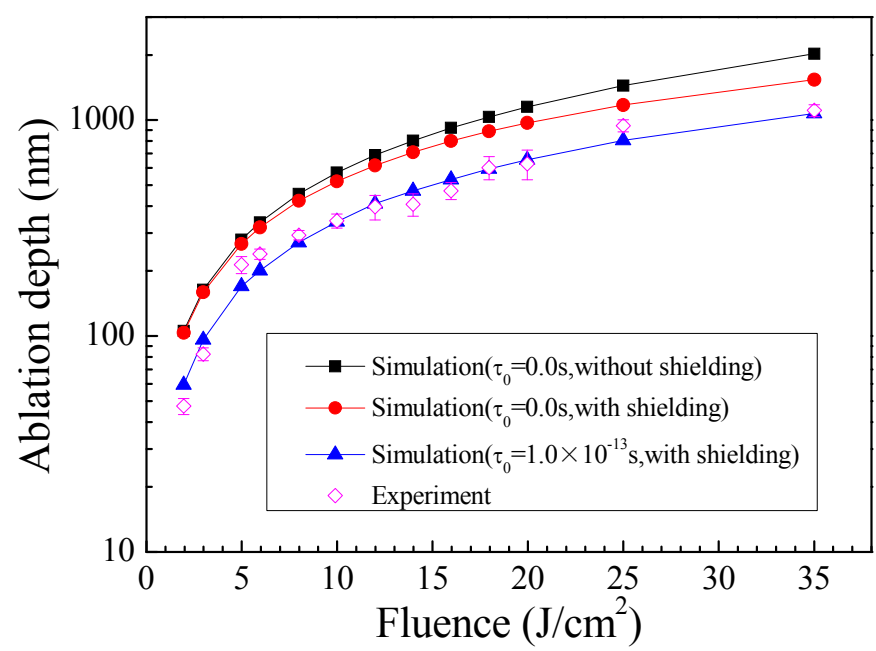

Figure 10. Comparison of simulation results of ablation depth with the experimental results from Reference [58].

\section{Conclusions}

The non-Fourier heat conduction model of ultra-short pulsed laser ablation of metal established in this paper takes into account the effect of heat source, laser heating of the target, the evaporation and phase explosion of target material, the formation and expansion of the plasma plume, and interaction of the plasma plume with the incoming laser. In addition, temperature dependent of optical and thermophysical properties are considered in the model due to the properties of the target will change over a wide range during the ultra-short pulsed laser ablation process. The results are summarized as follows:

1. The plasma shielding is an important physical mechanism that cannot be ignored in the process of ultra-short pulsed laser ablation, especially at high laser fluence.

2. The non-Fourier effect has a notable effect on the temperature characteristics and ablation depth of the target. The maintenance time of phase explosion decreases with the increase of relaxation time and the ablation depth is shallower with the increase of the relaxation time.

3. The ablation depth increases with the increase of the laser fluence and the biggest difference in ablation depth under different laser fluences is at the FWHM of the laser, and the difference of ablation depth caused by other time is small.

4. The ablation mechanism of ultra-short pulsed laser ablation is dominated by phase explosion and the ultra-short pulsed laser ablation can effectively reduce the heat affected zone compared to nanosecond pulsed laser ablation.

5. The comparison between the simulation results and the experimental results in literature shows that the model without considering the plasma shielding and the non-Fourier effect may result in overestimation of the ablation depth. On the contrary, the simulation results based on the model considering plasma shielding and the non-Fourier effect are in better agreement with the experimental results which indicates that the model with considering the plasma shielding and the non-Fourier effect can better simulate the ultra-short pulsed laser ablation process.

Author Contributions: S.T. provided ideas and methods for the research of this paper. J.W. and Y.Z. programmed and calculated the model. S.T. wrote the manuscript, and M.W. revised the manuscript. Y.O. provided useful suggestion during the whole work and helped polish the language of the manuscript.

Funding: This research was funded by National Natural Science Foundation of China (Grant number 11772354).

Conflicts of Interest: The authors declare no conflict of interest. 


\section{Nomenclature}

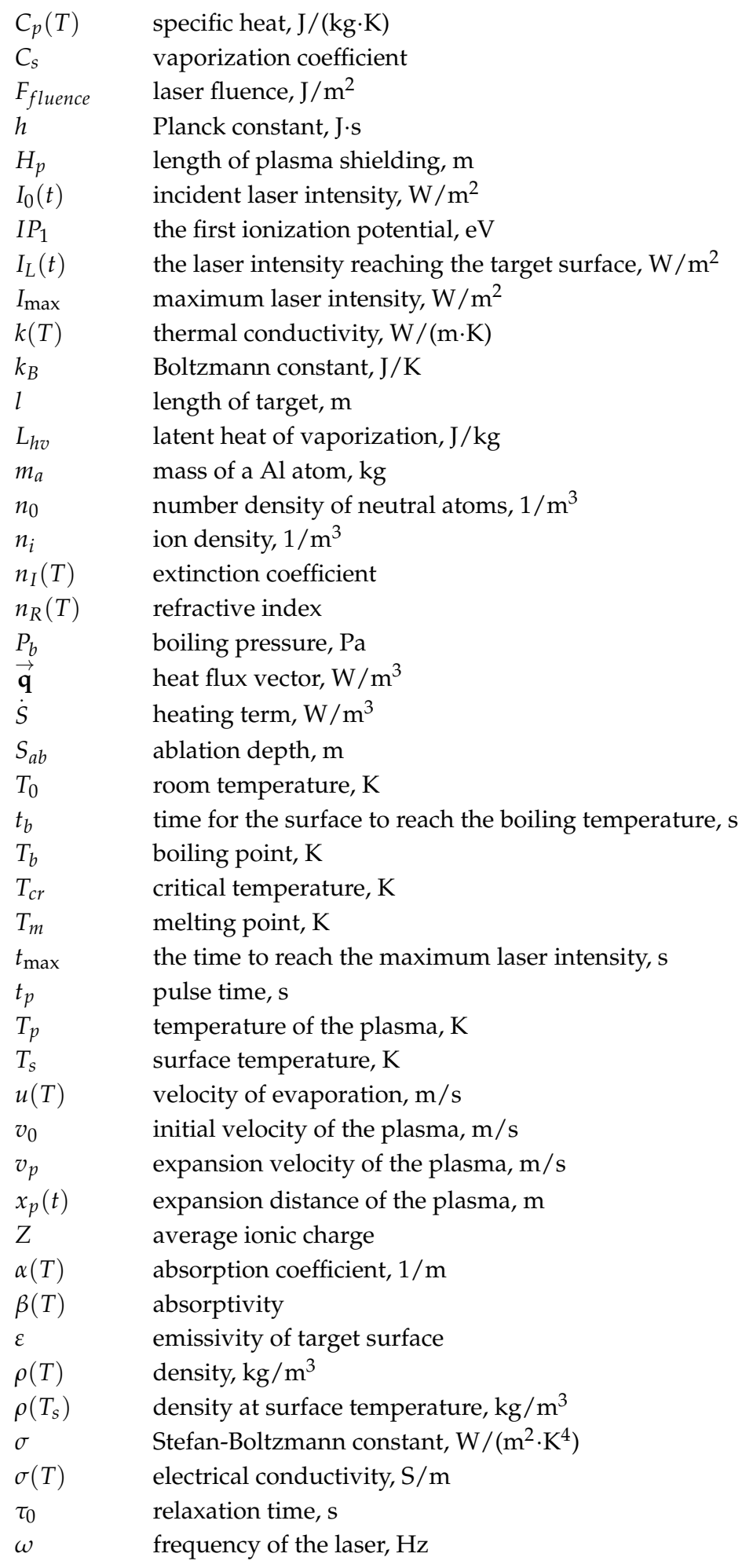

\section{References}

1. Shirk, D.; Molian, P.A. A review of ultrashort pulsed laser ablation of materials. J. Laser Appl. 1998, 10, 18-28. [CrossRef]

2. Mao, Y.D.; Xu, M.T. Non-Fourier heat conduction in a thin gold film heated by an ultra-fast-laser. Sci. China Technol. Sci. 2015, 58, 638-649. [CrossRef] 
3. Amoruso, S.; Ausanio, G.; Bruzzese, R.; Vitiello, M.; Wang, X. Femtosecond laser pulse irradiation of solid targets as a general route to nanoparticle formation in a vacuum. Phys. Rev. B 2005, 71, 033406. [CrossRef]

4. Tsakiris, N.; Anoop, K.K.; Ausanio, G.; Gill-Comeau, M.; Bruzzese, R.; Amoruso, S.; Lewis, L.J. Ultrashort laser ablation of bulk copper targets: Dynamics and size distribution of the generated nanoparticles. J. Appl. Phys. 2014, 115, 243301. [CrossRef]

5. Liu, Z.; Ning, F.; Cong, W.; Jiang, Q.; Li, T.; Zhang, H.; Zhou, Y. Energy consumption and saving analysis for laser engineered net shaping of metal powders. Energies 2016, 9, 763. [CrossRef]

6. Liebig, C.M.; Srisungsitthisunti, P.; Weiner, A.M.; Xu, X. Enhanced machining of steel using femtosecond pulse pairs. Appl. Phys. A 2010, 101, 487-490. [CrossRef]

7. Herman, P.R.; Oettl, A.; Chen, K.P.; Marjoribanks, R.S. Laser micromachining of transparent fused silica with 1-ps pulses and pulse trains. In Proceedings of the SPIE-The International Society for Optical Engineering, San Jose, CA, USA, 4 June 1999. [CrossRef]

8. Derrien, T.J.; Krüger, J.; Itina, T.E.; Höhm, S.; Rosenfeld, A.; Bonse, J. Rippled area formed by surface plasmon polaritons upon femtosecond laser double-pulse irradiation of silicon. Opt. Express 2014, 117, 77-81. [CrossRef] [PubMed]

9. Tan, S.; Wu, J.; Zhang, Y.; Cheng, Y.; Li, J.; Ou, Y. Research progress of laser-supported space micropropulsion technology. J. Propuls. Technol. 2018, 39, 2415-2428. (In Chinese)

10. Piñon, V.; Fotakis, C.; Nicolas, G.; Anglos, D. Double pulse laser-induced breakdown spectroscopy with femtosecond laser pulses. Spectrochim. Acta Part B 2008, 63, 1006-1010. [CrossRef]

11. Miyamoto, I.; Horn, A.; Gottmann, J.; Wortmann, D.; Yoshino, F. Fusion welding of glass using femtosecond laser pulses with high-repetition rates. J. Laser Micro Nanoeng. 2007, 2, 57-63. [CrossRef]

12. Cao, Y.; Zhao, X.; Shin, Y.C. Analysis of nanosecond laser ablation of aluminum with and without phase explosion in air and water. J. Laser Appl. 2013, 25, 032002. [CrossRef]

13. Wang, Y.; Shen, N.; Befekadu, G.K.; Pasiliao, C.L. Modeling pulsed laser ablation of aluminum with finite element analysis considering material moving front. Int. J. Heat Mass Transf. 2017, 113, 1246-1253. [CrossRef]

14. Zhang, Y.; Tzou, D.Y.; Chen, J.K. Micro- and Nanoscale Heat Transfer in Femtosecond Laser Processing of Metals. In High-Power and Femtosecond Lasers: Properties, Materials and Applications, 1st ed.; Nova Science Publisher: New York, NY, USA, 2009; pp. 159-206.

15. Qi, H.T.; Xu, H.Y.; Guo, X.W. The Cattaneo-type time fractional heat conduction equation for laser heating. Comput. Math. Appl. 2013, 66, 824-831. [CrossRef]

16. Rahideh, H.; Malekzadeh, P.; Haghighi, M.R.G. Non-fourier heat conduction analysis with temperature-dependent thermal conductivity. ISRN Mech. Eng. 2011, 321605. [CrossRef]

17. Zhang, L.; Shang, X. Analytical solution to non-Fourier heat conduction as a laser beam irradiating on local surface of a semi-infinite medium. Int. J. Heat Mass Transf. 2015, 85, 772-780. [CrossRef]

18. Catteneo, C. A form of heat conduction equation which eliminates the paradox of instantaneous propagation. Compte Rendus 1958, 247, 431-433.

19. Vernotte, P. Les paradoxes de la theorie continue de l'equation de la chaleur. Compte Rendus 1958, 246, 3154-3155.

20. Vick, B.; ÖZisik, M.N. Growth and decay of a thermal pulse predicted by the hyperbolic heat conduction equation. J. Heat Transf. 1983, 105, 902-907. [CrossRef]

21. Li, S.N.; Cao, B.Y. Mathematical and information-geometrical entropy for phenomenological Fourier and non-Fourier heat conduction. Phys. Rev. E 2017, 96, 032131. [CrossRef] [PubMed]

22. Tang, D.W.; Araki, N. Non-Fourier heat conduction behavior in finite mediums under pulsed surface heating. Mater. Sci. Eng. A 2000, 292, 173-178. [CrossRef]

23. Tzou, D.Y. Experimental support for the lagging behavior in heat propagation. J. Thermophys. Heat Transf. 1995, 9, 686-693. [CrossRef]

24. Tzou, D.Y.; Chiu, K.S. Temperature-dependent thermal lagging in ultrafast laser heating. Int. J. Heat Mass Transf. 2001, 44, 1725-1734. [CrossRef]

25. Zhang, D.; Li, L.; Li, Z.; Guan, L.; Tan, X. Non-Fourier conduction model with thermal source term of ultra short high power pulsed laser ablation and temperature evolvement before melting. Phys. B Condens. Matter 2005, 364, 285-293. [CrossRef]

26. Chen, T.M. A hybrid Green's function method for the hyperbolic heat conduction problems. Int. J. Heat Mass Transf. 2009, 52, 4273-4278. [CrossRef] 
27. Ahmadikia, H.; Moradi, A.; Fazlali, R.; Parsa, A.B. Analytical solution of non-Fourier and Fourier bioheat transfer analysis during laser irradiation of skin tissue. J. Mech. Sci. Technol. 2012, 26, 1937-1947. [CrossRef]

28. Kumar, S.; Bag, S.; Baruah, M. Finite element model for femtosecond laser pulse heating using dual phase lag effect. J. Laser Appl. 2016, 28, 032008. [CrossRef]

29. Noroozi, M.J.; Saedodin, S.; Ganji, D.D. A new solution for nonlinear dual phase lagging heat conduction problem. Alex. Eng. J. 2016, 55, 1745-1752. [CrossRef]

30. Ma, J.; Sun, Y.; Yang, J. Analytical solution of non-Fourier heat conduction in a square plate subjected to a moving laser pulse. Int. J. Heat Mass Transf. 2017, 115, 606-610. [CrossRef]

31. Soares, D. An enhanced explicit technique for the solution of non-Fourier heat transfer problems. Adv. Eng. Softw. 2018, 122, 13-21. [CrossRef]

32. Chichkov, B.N.; Momma, C.; Nolte, S.; von Alvensleben, F.; Tünnermann, A. Femtosecond, picosecond and nanosecond laser ablation of solids. Appl. Phys. A 1996, 63, 109-115. [CrossRef]

33. Wu, B.; Shin, Y.C. A simple model for high fluence ultra-short pulsed laser metal ablation. Appl. Surf. Sci. 2007, 253, 4079-4084. [CrossRef]

34. Cheng, J.; Perrie, W.; Wu, B.; Tao, S.; Edwardson, S.P.; Dearden, G.; Watkins, K.G. Ablation mechanism study on metallic materials with a 10 ps laser under high fluence. Appl. Surf. Sci. 2009, 255, 8171-8175. [CrossRef]

35. Hu, W.; Shin, Y.C.; King, G. Modeling of multi-burst mode pico-second laser ablation for improved material removal rate. Appl. Phys. A 2010, 98, 407-415. [CrossRef]

36. Qiu, T.Q.; Tien, C.L. Femtosecond laser heating of multi-layer metals-I. Analysis. Int. J. Heat Mass Transf. 1994, 37, 2789-2797. [CrossRef]

37. Tzou, D.Y.; Chen, J.K.; Beraun, J.E. Recent development of ultrafast thermoelasticity. J. Therm. Stress. 2005, 28, 563-594. [CrossRef]

38. Singh, N. Two-temperature model of non-equilibrium electron relaxation: A review. Int. J. Mod. Phys. B 2010, 24, 1141-1158. [CrossRef]

39. Qiu, T.Q.; Tien, C.L. Heat transfer mechanisms during short-pulse laser heating of metals. J. Heat Tranf. 1993, 115, 835-841. [CrossRef]

40. Chen, J.K.; Beraun, J.E. Numerical study of ultrashort laser pulse interactions with metal films. Numer. Heat Transf. Part A Appl. 2001, 40, 1-20. [CrossRef]

41. Jiang, L.; Tsai, H.L. Improved two-temperature model and its application in ultrashort laser heating of metal films. J. Heat Transf. 2005, 127, 1167-1173. [CrossRef]

42. Chen, J.K.; Tzou, D.Y.; Beraun, J.E. A semiclassical two-temperature model for ultrafast laser heating. Int. J. Heat Mass Transf. 2006, 49, 307-316. [CrossRef]

43. Carpene, E. Ultrafast laser irradiation of metals: Beyond the two-temperature model. Phys. Rev. B 2006, 74, 024301. [CrossRef]

44. Fang, R.; Wei, H.; Li, Z.; Zhang, D. Improved two-temperature model including electron density of states effects for Au during femtosecond laser pulses. Solid State Commun. 2012, 152, 108-111. [CrossRef]

45. Zhang, J.; Chen, Y.; Hu, M.; Chen, X. An improved three-dimensional two-temperature model for multi-pulse femtosecond laser ablation of aluminum. J. Appl. Phys. 2015, 117, 063104. [CrossRef]

46. Shin, T.; Teitelbaum, S.W.; Wolfson, J.; Kandyla, M.; Nelson, K.A. Extended two-temperature model for ultrafast thermal response of band gap materials upon impulsive optical excitation. J. Chem. Phys. 2015, 143, 194705. [CrossRef] [PubMed]

47. Qiu, T.Q.; Juhasz, T.; Suarez, C.; Bron, W.E.; Tien, C.L. Femtosecond laser heating of multi-layer metals-II. Experiments. Int. J. Heat Mass Transf. 1994, 37, 2799-2808. [CrossRef]

48. Ciegis, R.; Dement'ev, A.; Jankeviciute, G. Numerical analysis of the hyperbolic two-temperature model. Lith. Math. J. 2008, 48, 46-60. [CrossRef]

49. Zhou, L.; Li, L. Melting and resolidification of gold film irradiated by laser pulses less than $100 \mathrm{fs}$. Appl. Phys. A 2014, 116, 2157-2165. [CrossRef]

50. Majchrzak, E.; Dziatkiewicz, J. Analysis of ultrashort laser pulse interactions with metal films using a two-temperature model. J. Appl. Math. Comput. Mech. 2015, 14, 31-39. [CrossRef]

51. Li, L.; Zhao, S. Thermal ablation of thin gold films irradiated by ultrashort laser pulses. Appl. Phys. A 2016, 122, 436. [CrossRef]

52. Dziatkiewicz, J.; Majchrzak, E. Numerical analysis of laser ablation using the axisymmetric two-temperature model. AIP Conf. Proc. 2018, 1922, 060004. [CrossRef] 
53. Hakkinen, H.; Landman, U. Superheating, melting, and annealing of copper surfaces. Phys. Rev. Lett. 1993, 71, 1023-1026. [CrossRef] [PubMed]

54. Ivanov, D.S.; Zhigilei, L.V. Combined atomistic-continuum modeling of short-pulse laser melting and disintegration of metal films. Phys. Rev. B 2003, 68, 064114. [CrossRef]

55. Sonntag, S.; Roth, J.; Gaehler, F.; Trebin, H.R. Femtosecond laser ablation of aluminium. Appl. Surf. Sci. 2009, 255, 9742-9744. [CrossRef]

56. Ji, P.; Zhang, Y. Melting and thermal ablation of a silver film induced by femtosecond laser heating: A multiscale modeling approach. Appl. Phys. A 2017, 123, 671. [CrossRef]

57. Colombier, J.P.; Combis, P.; Bonneau, F.; Le Harzic, R.; Audouard, E. Hydrodynamic simulations of metal ablation by femtosecond laser irradiation. Phys. Rev. B 2005, 71, 165406. [CrossRef]

58. Davydov, R.V.; Antonov, V.I. Simulation of femtosecond pulsed laser ablation of metals. J. Phys. Conf. Ser. 2016, 769, 012060. [CrossRef]

59. Taylor, L.L.; Scott, R.E.; Qiao, J. Integrating two-temperature and classical heat accumulation models to predict femtosecond laser processing of silicon. Opt. Mater. Express 2018, 8, 648-658. [CrossRef]

60. Fann, W.S.; Storz, R.; Tom, H.W.K.; Bokor, J. Direct measurement of nonequilibrium electron-energy distributions in subpicosecond laser-heated gold films. Phys. Rev. Lett. 1992, 68, 2834-2837. [CrossRef] [PubMed]

61. Groeneveld, R.H.M.; Sprik, R.; Lagendijk, A. Femtosecond spectroscopy of electro-electron and electron-phonon energy relaxation in Ag and Au. Phys. Rev. B 1995, 51, 11433. [CrossRef]

62. Schmidt, V.; Husinsky, W.; Betz, G. Ultrashort laser ablation of metals: Pump-probe experiments, the role of ballistic electrons and the two-temperature model. Appl. Surf. Sci. 2002, 197-198, 145-155. [CrossRef]

63. Byskov-Nielsen, J.; Savolainen, J.-M.; Christensen, M.S.; Balling, P. Ultra-short pulse laser ablation of copper, silver and tungsten: Experimental data and two-temperature model simulations. Appl. Phys. A 2011, 103, 447-453. [CrossRef]

64. Christensen, B.H.; Vestentoft, K.; Balling, P. Short-pulse ablation rates and the two-temperature model. Appl. Surf. Sci. 2007, 253, 6347-6352. [CrossRef]

65. Abdelmalek, A.; Bedrane, Z.; Amara, E. Thermal and non-thermal explosion in metals ablation by femtosecond laser pulse: Classical approach of the two temperature model. J. Phys. Conf. Ser. 2018, 987, 012012. [CrossRef]

66. Porneala, C.; Willis, D.A. Effect of the dielectric transition on laser-induced phase explosion in metals. Int. J. Heat Mass Transf. 2006, 49, 1928-1936. [CrossRef]

67. Zhang, Y.; Zhang, D.; Wu, J.; He, Z.; Deng, X. A thermal model for nanosecond pulsed laser ablation of aluminum. AIP Adv. 2017, 7, 075010. [CrossRef]

68. Marla, D.; Bhandarkar, U.V.; Joshi, S.S. Critical assessment of the issues in the modeling of ablation and plasma expansion processes in the pulsed laser deposition of metals. J. Appl. Phys. 2011, 109, 021101. [CrossRef]

69. Zhang, Z.; Liu, D.Y. Liu, Advances in the study of non-Fourier heat conduction. Adv. Mech. 2000, 30, 446-456. (In Chinese)

70. Peterlongo, A.; Miotello, A.; Kelly, R. Laser-pulse sputtering of aluminum: Vaporization, boiling, superheating, and gas-dynamic effects. Phys. Rev. E 1994, 50, 4716-4727. [CrossRef]

71. Gragossian, A.; Tavassoli, S.H.; Shokri, B. Laser ablation of aluminum from normal evaporation to phase explosion. J. Appl. Phys. 2009, 105, 103304. [CrossRef]

72. Marla, D.; Bhandarkar, U.V.; Joshi, S.S. A model of laser ablation with temperature-dependent material properties, vaporization, phase explosion and plasma shielding. Appl. Phys. A 2014, 116, 273-285. [CrossRef]

73. Singh, R.K.; Narayan, J. Pulsed-laser evaporation technique for deposition of thin films-physics and theoretical model. Phys. Rev. B 1990, 41, 8843-8859. [CrossRef]

74. Chen, F.F. Introduction to Plasma Physics and Controlled Fusion, 2st ed.; Volume 1: Plasma Physics, Plenum Press: New York, NY, USA, 1985; pp. 1-5.

75. Garrelie, F.; Aubreton, J.; Catherinot, A. Monte carlo simulation of the laser-induced plasma plume expansion under vacuum: Comparison with experiments. J. Appl. Phys. 1998, 83, 5075-5082. [CrossRef]

76. Lide, D.R.; Haynes, W.M. CRC Handbook of Chemistry and Physics, 90th ed.; CRC Press: Boca Raton, FL, USA, 2010 . 
77. Wu, B.; Shin, C.Y. Absorption coefficient of aluminum near the critical point and the consequences on high-power nanosecond laser ablation. Appl. Phys. Lett. 2006, 89, 111902. [CrossRef]

78. Desai, P.D.; James, H.M.; Ho, C.Y. Electrical resistivity of aluminum and manganese. J. Phys. Chem. Ref. Data 1984, 13, 1131-1172. [CrossRef] 